\title{
La enseñanza del español como lengua materna. Presupuestos teóricos-metodológicos
}

\author{
Fecha de recepción : 2020-04-08 • Fecha de aceptación: 2020-06-22 • Fecha de publicación: 2020-09-10
}

Armando Castillo Acevedo ${ }^{1}$

Instituto Preuniversitario Urbano: Miguel Diosdado Pérez Pimentel, Cuba

armandojamil26@yahoo.com

https://orcid.org/0000-0002-3300-9031

Heriberto Nuñez Rodríguez ${ }^{2}$

EPP: Manuel Ascunce Domenech, Cuba

heri23@nauta.cu

https://orcid.org/0000-0001-6648-1570

\section{Resumen}

Los estudios más recientes de la enseñanza del español, como lengua materna, se han desplazado hacia situaciones comunicativas variadas, donde el hablante demuestra sus capacidades lingüísticas y sus particularidades como ente social que actúa -y por tanto habla- en dependencia de la situación comunicativa a la que esté expuesto. Es innegable que el espacio ideal para la correcta aprensión de la lengua es la escuela, pues el estudiante no solo perfecciona el dominio de la lengua oral que aprendió de sus padres, en un medio sociocultural determinado; sino que se apropia de los códigos de la lengua escrita y las normas que rigen su uso. Este artículo tiene como objetivo analizar la situación actual de la enseñanza del español como lengua materna; para ello se presentan los fundamentos teóricos y metodológicos que sustentan dicho proceso y algunos instrumentos didácticos que contribuirán a su perfeccionamiento. La enseñanza de la lengua debe verse como un proceso integrador, que aborde desde la relación entre significante y significado hasta su variación en diferentes contextos, a fin de que la enseñanza - aprendizaje del español como lengua materna sea fructífero, y logre ese carácter bilateral que debe poseer una clase de lengua de excelencia. 
Palabras clave: foro virtual, aula interactiva, universidad, nivel de ajuste curricular.

\begin{abstract}
The most recent studies of the teaching of spanish as a mother tongue have moved towards various communicative situations, where the speaker demonstrates his linguistic capacities and his particularities as a social entity, which acts -and therefore speaks- depending on the communicative situation. the one that is exposed. It is undeniable that the ideal space for the correct learning of the language is the school, since the student not only perfects the command of the oral language that he learned from his parents, in a certain sociocultural environment; rather, it appropriates the codes of the written language and the norms that govern its use. This article aims to analyze the current situation of teaching spanish as a mother tongue; for this, it is explained the theoretical and methodological foundations that support this process and some didactic instruments that will contribute to its improvement. The teaching of the language must be seen as an integrating process, which covers from the relationship between the signifier and the signified to its variation in different contexts, so that the teaching - learning of Spanish as a mother tongue is fruitful, and achieves that bilateral character that You must have a language class of excellence.
\end{abstract}

Keywords: mother tongue, teaching - learning, spanish, students, teachers. 


\section{Introducción}

Para llevar a cabo el estudio de la enseñanza del español como lengua materna es preciso tener en cuenta los aportes que ha hecho la psicolingüística, semiótica, sociolingüística y la pragmática en lo referente a la comunicación social en una comunidad de hispanohablantes. Los especialistas de dichas disciplinas se han desplazado hacia nuevos horizontes, donde podrán reflexionar sobre el funcionamiento del lenguaje en diversas situaciones comunicativas y la actividad lingüística de los hablantes, atendiendo a las particularidades socio-culturales y lingüísticas de los mismos.

La enseñanza de la lengua tiene como finalidad incrementar y perfeccionar progresivamente en el ambiente escolar el aprendizaje natural y espontáneo que el alumno realiza de la lengua oral en su ambiente sociolingüístico, así como la adquisición de la lengua escrita con la guía del docente.

El objetivo general inmediato de la enseñanza lingüística consiste en poner un acto y perfeccionar las capacidades del alumno para la expresión y comprensión verbal, en sus manifestaciones oral y escrita, mientras que el objetivo mediato es la formación intelectual, dada por la íntima relación que existe entre lenguaje y pensamiento, reflejada por la naturaleza misma del signo lingüístico (significante - significado).

El presente trabajo tiene como objetivo analizar la situación actual de la enseñanza del español como lengua materna; para ello se ofrecen los fundamentos teóricos y metodológicos que sustentan el proceso de enseñanza - aprendizaje; además de algunos instrumentos didácticos que contribuyan al perfeccionamiento de la clase de lengua, tales como: atención a los alumnos con dificultades en la aprensión de la lengua, tareas individualizadas, trabajo independiente para el hogar, relacionar el contenido que se está impartiendo con el medio sociocultural en el que convive, juegos didácticos, proyección de audiovisuales que aborden el uso correcto de la lengua, fomentar la lectura a través de talleres u otra variante que asuma el docente atendiendo siempre al diagnóstico de sus alumnos.

\section{Presupuestos teórico - metodológicos de la enseñanza de la lengua}

Es sabido que a través del uso diario del lenguaje el ser humano va creando conciencia del mundo que lo rodea. La lengua está considerada como el intermedio necesario e imprescindible entre los hombres, y estos con la realidad; la percepción e interpretación de dicha realidad dependen de la palabra de forma decisiva.

Después de Saussure (1975), la lengua se ha definido como ese sistema de signos y reglas. Es por eso que la lingüística se ha encargado de su estudio profundo sin tener en cuenta el uso lingüístico de los hablantes o el contexto en el que se producen tanto la comunicación oral, como la escrita.

Los didactas no pueden perder de vista que la enseñanza de la lengua no es solo reconocer sílabas, morfemas, oraciones de manera aislada o la enseñanza de las normas que rigen el idioma, sino que "... la lengua es para usarla, para hacer posible establecer contactos con otros, para lograr el desarrollo cognoscitivo y social de los hombres como instrumento esencial e 
imprescindible en el (...) proceso de aprendizaje y conocimiento de la realidad..." (Bruzal, 2007, p.6).

Es importante centrar el estudio en las unidades discursivas, o sea, no limitarlo al estrecho marco de la palabra y la oración, ya que los estudiantes elaboran sus actos comunicativos más allá de estas.

En lo lingüístico debemos tener en cuenta que el código oral y escrito son modalidades distintas pero que tienen un mismo objetivo: una comunicación eficaz y el desarrollo del lenguaje. Por tanto, debe evitarse que los rasgos fonéticos del habla de los estudiantes no se reflejen en el proceso de la escritura, ya que "no se debe escribir como se habla, ni hablar como se escribe" (Bruzal, 2007, p. 6)

La lengua materna se entiende como la madre, tanto del medio familiar como del socio-cultural; esta no influye únicamente en el modo en que el ser humano manifiesta sus reacciones frente el mundo que lo rodea, sino que estructura la manera en que este percibe el mundo. Por tanto, podemos denominar lengua materna a aquella que, durante la infancia, el niño aprende de su familia en un medio social determinado.

De esta manera, el docente tiene como obligación "....atender el desarrollo en los alumnos de la reflexión sobre la lengua materna, lo cual incluye la reflexión gramatical y la comprensión y producción textual, que deben estar estrechamente ligadas; también los aspectos de la comunicación en cuanto a lo verbal y lo no verbal..." (Bruzal, 2007, p.6). Dicho esto, es preciso señalar que la enseñanza de la lengua materna debe realizarse de forma contextual, para que el estudiante sea capaz de ver las variaciones socio-lingüísticas que tiene el idioma. Por lo que " $\ldots$ al aprender a usar una lengua no solo aprendemos a construir frases gramaticalmente correctas, sino también a saber qué decir, a quién, cuándo y cómo decirlo y qué y cuándo callar" (Lomas, 1999, p.162).

Lo expuesto con anterioridad determinan para el área de la lengua y literatura un enfoque funcional comunicativo que difiere de los esquemas tradicionales, centrados en la teoría gramatical, el historicismo y el formalismo literario que atiende al desarrollo de la competencia comunicativa del alumno, entendida no sólo como el conocimiento del sistema lingüístico y los códigos no verbales, sino también de la adecuación de su actuación lingüística a los diferentes contextos y situaciones comunicativas (Ministerio de Educación, Cultura y Deporte, 1997).

En cuanto al desarrollo cognitivo del estudiante, durante el proceso de aprendizaje del español como lengua materna no puede olvidarse que "el proceso de adquirir y aprender una lengua se realiza en el ser humano a partir de actos comunicativos en situaciones comunicativas. Y esto puede lograrse a través de la percepción" (Bruzal 2007, p.9). Por lo que el estudiante es capaz de percibir y representar los significados antes que el significante, y a partir de esto comienza a relacionar los significados con los grupos fónicos que domina desde la infancia.

Según los estudios de Bruzal (2007), lo primero que el estudiante debe aprender en el contexto escolar es leer y escribir su lengua, en este caso el español, puesto que esa es la lengua que 
conoce. Vale destacar que no se debe comenzar a enseñar la lengua materna a partir de un proceso memorístico de reglas gramaticales, ya que lo primero que el alumno debe comprender es el sentido y significado de los mensajes que recibe constantemente, tanto fuera como dentro del ámbito de la escuela (p. 11).

\section{Breve caracterización de la enseñanza del español como lengua materna}

La enseñanza del español como lengua materna, a todos los niveles, ha tenido como fin que el estudiante sea capaz de hacer uso del idioma que aprendió de sus padres correctamente, o sea, respetando las normas cultas del español que se habla en el país y bajo la dirección y guía del profesor de esta materia; por lo que se ha transitado por diferentes momentos y modificaciones en los planes de estudio.

Desde el preescolar, con un carácter propedéutico, se hace énfasis en la enseñanza de los sonidos, la correcta articulación y pronunciación de determinados fonemas que deberán estar acordes a su desarrollo psicológico, además de los primeros rasgos, con el fin de acercarlos a las normas caligráficas que posteriormente, a partir del primer grado de la enseñanza primaria, deberán conocer y aplicar.

En este nivel, la asignatura permite que el alumno tenga un mejor acercamiento a su lengua materna, desde dominar el código escrito y la lectura, hasta lograr que sean capaces de redactar textos con fines y propósitos determinados, además de la correcta comprensión textual, siempre atendiendo al nivel en que se encuentra el educando.

En su artículo "Los enfoques comunicativos: elogio y crítica" el didacta barcelonés Daniel Cassany (1994) hace una alerta sobre la necesidad de no dejar a la literatura aislada de la enseñanza de la lengua: “(...) En cambio, desde sus inicios, las propuestas comunicativas conectaron directamente con las necesidades sociales, laborales o académicas del alumnado, prescindiendo de las esferas artísticas de la cultura (y la literatura)".

Puesto que lengua y literatura no pueden tratarse por separados, los planes de estudio más recientes y el método discursivo funcional que propone la Roméu (2007) posibilitan que el alumno pueda apoyarse en los textos literarios, como un referente en el perfeccionamiento de su lengua materna, estos sirven de modelo para el estudiante en la asimilación de la norma culta.

El alumnado de las enseñanzas media y media superior deberá poner especial atención al uso de la lengua en las obras literarias estudiadas durante las clases de lengua y literatura, sobre todo en los escritos originalmente en español, debido a que estas son las que van a contribuir concretamente a que el estudiante conozca la evolución que ha tenido nuestra lengua de forma diacrónica, y a que haya un notable enriquecimiento de su vocabulario, de acuerdo a su desarrollo psicológico y fisiológico.

Este estrecho vínculo entre la lengua y la literatura influye de manera positiva en el desarrollo de las habilidades generales y específicas, propias de la asignatura ya mencionada. La ejercitación 
de los contenidos lingüísticos durante el análisis literario favorece la frecuente práctica verbal de los alumnos para exponer sus conocimientos y opiniones, lo que redunda satisfactoriamente en el nivel de expresión que alcanzan y en la forma independiente en que logran organizar su pensamiento.

"Mientras la enseñanza tradicional ponía el énfasis en la acumulación de datos y concebía al alumno como un banco donde se depositaban conocimientos, en la actualidad se considera como un ente activo, capaz de participar conscientemente en la adquisición de nuevos conocimientos, autorregular sus aprendizajes, atribuirle significatividad a lo que aprende y estar motivado intrínseca y extrínsecamente por aprender. Hoy la enseñanza - aprendizaje de la lengua (...) se propone lograr que los estudiantes se conviertan en comunicadores eficientes, para lo cual es necesario que posean conocimientos acerca del sistema de la lengua y su funcionamiento en el discurso, teniendo en cuenta la diversidad de tipos de discursos que el individuo emplea en los diferentes contextos en los que interactúa" (Roméu, 2013, p.2).

Por lo anteriormente expuesto es que los modelos constructivistas y el enfoque cognitivo, comunicativo y sociocultural para la enseñanza de la lengua que hoy se persigue en las instituciones educativas, favorecen una asimilación del conocimiento más efectiva, basada en la independencia cognoscitiva del estudiante, ya sea dentro o fuera del ambiente escolar.

En función de lograr habilidades en la aprehensión de la lengua y la literatura, los componentes funcionales de la clase (comprensión, construcción y análisis) deben concretarse en los ejes de organización de los contenidos: literatura, estructuras lingüísticas en uso, normativa y lenguaje y comunicación, además de dar una atención priorizada al correcto uso de las macrohabilidades (escuchar, leer, escribir, hablar), y de este modo contribuir al desarrollo de destrezas en los estudiantes durante el proceso de enseñanza - aprendizaje del español y en el empleo del lenguaje como medio de cognición y comunicación entre el alumno y el profesor.

Los docentes deberán insistir en la lectura y relectura de textos literarios y no literarios ya que solo a partir de esto serán capaces de comprender las diversas formas que puede adoptar la lengua, ya que esta depende de la tipología textual, el contexto, el estrato social representado e incluso, las características propias del escritor, como exigencia de la enseñanza en la escuela cubana actual (Roméu, 2007). A partir del análisis de los elementos necesarios sobre la lengua en función de la literatura, esta materia contribuirá en los estudiantes a la formación de una concepción científica del mundo y a un desarrollo del pensamiento lógico y coherente, atendiendo siempre a sus necesidades actuales y particulares.

Los estudios han demostrado que la enseñanza de la lengua en la escuela actual puede tener un carácter interdisciplinario, multidisciplinario y transdisciplinario puesto que todas las materias que se imparten en las instituciones educativas se valen de los recursos lingüísticos que brinda el español, ya sea como medio para la adquisición del conocimiento o para comunicarse. Como ya se ha dicho, la lengua es el macroeje transversal, tanto de los profesores de español y literatura, como de los de otras materias y niveles, por lo que esto los convierte, de algún modo, en profesores de lengua. 
El aporte de las demás disciplinas a la enseñanza del español, como lengua materna, tiene la base en su contribución a la comprensión y construcción de un pensamiento lógico y correctamente relacionado, además de la capacidad comunicativa de los estudiantes, en cualquier contexto social.

Hoy su enseñanza tiene dentro de sus objetivos generales que en los estudiantes se forme un sistema de conocimientos lingüísticos que les serán imprescindibles en su desarrollo como ente social, además, de una organización adecuada del pensamiento, según el grado o enseñanza por que esté transitando en ese momento. Por lo que dicha asignatura le facilita al estudiante las herramientas necesarias para la aplicación de los recursos que brinda la lengua durante el proceso de enseñanza - aprendizaje del idioma como lengua materna y su utilización en función de un desarrollo científico y literario dentro de la enseñanza a la cual pertenezca.

\section{La clase de español - literatura como espacio ideal para la enseñanza como lengua materna}

La clase de lengua, según Parra (1989), “... debe ser un contexto de interacción lingüística permanente, entre el maestro y los alumnos, para estudiar el lenguaje como dimensión de lo humano, estrechamente ligado a la vida y a la acción social del hombre" (Citado en Roméu, 2013, p. 62).

Atendiendo a lo expresado por Marina Parra, ciertamente el espacio ideal para que lo expuesto en el epígrafe anterior se materialice, es a través de una clase de excelencia. Una vez que el docente tenga planificada la clase de determinados contenidos lingüísticos o literarios, como el medio ideal para que se logre entre los estudiantes una comunicación libre de interferencias; no deberá dejar de lado las macrohabilidades (escribir, escuchar, hablar y leer).

Una clase de lengua alcanzará excelencia cuando en ella se le ofrezca la prioridad requerida a la comprensión, tanto del texto científico como del literario, para que de este modo el estudiante sea capaz de lograr la producción de significados. Se deberán crear situaciones comunicativas complejas para permitir al alumno que asuma nuevos roles comunicativos y se haga más independiente en el proceso, siempre en correspondencia con el nivel en que se encuentre. El profesor será el encargado de sistematizar el análisis de textos literarios y no literarios, en dependencia de las propuestas que hagan los planes de estudio en las distintas enseñanzas y del diagnóstico que posea cada profesor de su masa estudiantil; de esta manera los alumnos serán capaces de trabajar con los diferentes niveles de la lengua y hacer uso correcto de ellos durante la construcción de nuevos textos.

Lo mencionado está fundamentado en las concepciones de la lengua, en forma de macroeje y como medio esencial del conocimiento y la comunicación, por lo que se tendrá en cuenta durante la clase como el mejor medio para una comunicación sin interferencias que los alumnos adquieran de forma sistemática todos los elementos cognitivos de la lengua española, la forma de usarla en los diferentes discursos y contextos, siempre ajustándose a lo establecido por la norma culta, 
además de permitirle participar, de forma independiente, en la solución de las tareas comunicativas que se le propongan durante la clase de lengua.

Para Roméu (2013), las capacidades que se aspiran a desarrollar en las clases de EspañolLiteratura descansan en los conocimientos y habilidades que los alumnos deberán adquirir durante el proceso de enseñanza aprendizaje; suponer un alto grado de independencia en la solución de las tareas y la posibilidad de que los estudiantes lleguen a autorregular los procesos de comprensión, análisis y construcción, además de la capacidad para la creatividad durante la creación de textos literarios y no literarios, y luego desarrollar la autoevaluación.

El proceso de enseñanza - aprendizaje de la lengua y la literatura, como en el resto de las materias, se centra en el estudiante al que se le considera un sujeto activo en la búsqueda del conocimiento. Es por eso que durante este proceso deben tenerse en cuenta una serie de principios didácticos para dicho proceso: la cientificidad, la sistematización, la relación intermaterias, el aprendizaje educativo, la asimilación activa y consciente por parte de los estudiantes, la accesibilidad y asequibilidad, consolidación y solidez, el carácter individualizador y diferenciado y la vinculación de la teoría con la práctica; los cuales se vuelven imprescindibles para lograr lo deseado por el docente durante la clase.

Tampoco se debe dejar de la mano la relación entre los componentes funcionales de la lengua, así como la creación de situaciones complejas en el proceso comunicativo, con el propósito de hacer evidente la funcionalidad de los conocimientos adquiridos y la importancia que ha de concederle a la interdisciplinariedad.

A juicio de (Roméu, 1992) "Al buscar un elemento verdaderamente integrador de los contenidos de la clase de lengua, se llegó a la conclusión de que dicho elemento era la comunicación y los procesos de significación con ella relacionados. Dichos procesos eran: la compresión, el análisis y la construcción, que se denominan componentes funcionales" (Citado en Roméu, 2013, p. 43).

Es significativo que todo docente, durante la preparación de la clase de lengua, tenga en cuenta los componentes mencionados, ya que, aunque uno de ellos se convierta en componente rector durante la enseñanza de la lengua y la literatura, los otros dos estarán presentes como subordinados. Esto puede explicarse de la siguiente manera: en toda clase de español como lengua materna los componentes funcionales se combinan, lo que favorece un desarrollo fructífero del proceso de aprendizaje.

Para llegar a ser unos comunicadores competentes, los estudiantes deberán, primero que todo, aprender a comprender, construir y analizar textos de diferentes estilos y finalidades comunicativas. Cuando en la clase de lengua se les da tratamiento a estos componentes, el docente, junto con sus estudiantes, estarán sistematizando las habilidades lingüísticas: audición, lectura, habla y escritura.

Trabajar con los componentes funcionales en la enseñanza de español implica la jerarquización de uno sobre los otros. Esto exige que el docente conozca, según el objetivo trazado, cuál es el componente priorizado de la clase en cuestión. No significa esto que el resto de los componentes 
no estén presentes, ya que a la vez que se priorice uno, los otros se utilizarán de apoyo al trabajo con el componente priorizado.

En toda clase de lengua el desarrollo del conocimiento en el estudiantado estará vinculado a los componentes funcionales y al trabajo con ellos a partir de las estructuras que conforman los niveles de la lengua (fonológico, lexical, gramatical y textual). El profesor no ha de olvidar los contenidos que deberá trabajar durante el estudio de estos niveles, ya que solo de esa forma podrá contribuir al desarrollo intelectual de los alumnos. Se debe tener en cuenta que los conceptos y modos de actuar estén relacionados con aquellos elementos de identidad que encierra nuestra lengua y lo que nos hace poseedores de ella.

Cada estudiante tiene la posibilidad de adquirir y desarrollar habilidades y hábitos en las clases donde se enseña la lengua, su estructura y su función contextual. En estas clases se priorizarán las habilidades intelectuales, donde se revela la unidad existente entre el pensamiento y el lenguaje; las habilidades docentes: encaminadas a la toma de notas, elaboración de resúmenes, redacción, el comentario y la capacidad de autorregular dichos procesos, favorecen la actividad comunicativa en el aula, en el centro escolar o fuera de este. "Las habilidades constituyen acciones generales y específicas, mediante las cuales se manifiestan los contenidos" (Roméu, 2013, p. 16).

A medida que el alumno vaya cursando una enseñanza y posteriormente pase a una superior, será capaz de ir conociendo aquellas habilidades que tienen mayor cabida en las clases de lengua y literatura, al mismo tiempo que podrá ir desarrollándolas por sí solo y comprobar el desarrollo de sus conocimientos en esta materia. Dichas habilidades son: comprensión inteligente, crítica y creadora de los textos que serán objeto de estudio durante su tránsito por la enseñanza, uso estrategias para lograr comprender lo dicho, a través del uso de la lengua.

La lectura a viva voz y la declamación, la lectura comentada, y de otros tipos, se encuentran grandemente afectadas en la escuela actual, por el desinterés que muestran los estudiantes frente al texto literario o no literario y el poco hincapié que hacen muchos maestros. Esto está condicionado por el avance vertiginoso de las nuevas tecnologías de la información y las comunicaciones, las cuales ofrecen un producto elaborado desde la visión de otra persona y limita las posibilidades para el desarrollo de la imaginación que sí proporciona la lectura. Sin embargo, sigue siendo una obligación del docente demostrar a sus alumnos la marcada significación que tiene esta habilidad para el enriquecimiento de su vocabulario y el mejor dominio de su lengua materna.

Descubrir la regla y aplicarla en situaciones dadas para la copia consciente y la toma del dictado, está última muy afectada por el problema que hoy poseen nuestros estudiantes en la habilidad de la escucha. Lograr el análisis fonológico, morfológico, lexical, sintáctico, ortográfico, y textual de textos literarios o no literarios escritos originalmente en español, para poder observar, los distintos fines que se le dan a las estructuras lingüísticas en uso. La construcción de textos de diferentes tipos, estilos y funciones, teniendo en cuenta las dimensiones semánticas, pragmáticas, textuales sintácticas, además de ser capaces de redactar según sus intereses y haciendo uso de su propio código lingüístico, siempre respetando lo que establece la norma culta. El autocontrol y 
autocorrección del texto escrito, las habilidades para aprovechar las potencialidades educativas de los textos en función de la formación de valores y las convicciones.

El desarrollo de dichas habilidades, durante el proceso de enseñanza aprendizaje, favorecerá la fomentación de hábitos de la lectura, la búsqueda de información, la elaboración de fichas y sobre todo que los alumnos perfeccionen su modo de usar la lengua materna, además de hábitos para el trabajo con la ciencia y la creación literarias, siempre apoyándose de los recursos que ofrece a la literatura, el lenguaje.

En las clases de Español - Literatura, no solo se persigue el desarrollo de hábitos y habilidades, sino que se aspira, además, a desarrollar aquellas capacidades que el alumno ha adquirido en la asignatura, durante su paso por las todas las enseñanzas, las cuales se basarán en el alto grado de independencia durante la solución de tares docentes y su probabilidad de autorregular el trabajo con los componentes funcionales.

A juicio de Addine (2004), las capacidades no son más que “...el sistema de experiencias de la actividad creadora y es un contenido de mayor vuelo en el orden intelectual" (p. 71). Es por ello que el alumno será capaz de crear textos tanto literarios como científicos, a partir de la puesta en práctica de las habilidades adquiridas, en el proceso docente, lo cual le permite al estudiante “....independencia cognoscitiva, con el pensamiento de un desarrollo reflexivo y divergente y la imaginación creadora, entre otros" (Addine, 2004, p. 20). Evidenciándose en su capacidad para establecer un juicio crítico-valorativo acerca del texto objeto de análisis, sea literario o no, durante la clase, sino que evaluará a sus compañeros y será capaz de autoevaluar lo que escriba.

El uso y dominio de la lengua materna, como parte de nuestra identidad como hispanohablantes, se manifestará en el alumno según su actuación dentro y fuera del centro escolar de modo sistemático. Ya estos no solo serán el reflejo de lo que han aprendido en los hogares, sino que también serán la cara ante la sociedad de la labor del maestro de lengua, por lo que debe lograrse, desde la escuela, que el estudiante sea siempre un modelo lingüístico en cualquier contexto sociocultural.

\section{Metodología}

Para el desarrollo de este trabajo se asumió un enfoque cualitativo; ya que, para Hernández, Fernández \& Baptista (2014), este es específico y posibilita detallar las particularidades esenciales del tema objeto de investigación, además que permite precisar aquellos conceptos que se relacionan con dicha temática. Esta metodología contribuye a que los investigadores conozcan la realidad existente en el ámbito escolar respecto a la enseñanza del español como lengua materna. Además, se empleó el método analítico, el cual permite la desintegración del fenómeno en sus partes fundamentales a través de un estudio detallado, generando conocimientos nuevos que den lugar a conclusiones acertadas.

Por otra parte, es necesario precisar que los autores se apoyaron en la técnica bibliográfica 
- documental, puesto que para Hernández et al (2014), a través de esta se puede hacer una descripción, análisis e interpretación del tema de investigación, así como registrar la composición, procesos y naturaleza del fenómeno en investigado. Se llevó a cabo una revisión de elementos teóricos y metodológicos sobre la enseñanza del español como lengua materna, teniendo en cuenta tanto la bibliografía clásica como las trabajos más recientes, todo esto a través del manejo de libros, ensayos y artículos de revistas indexadas del ámbito didáctico y pedagógico. Esta técnica es la que posibilita el manejo de los conceptos y criterios especializados que sustentan el carácter científico del estudio. Lo anteriormente expuesto es un soporte fundamental para hacer comparaciones y arribar a conclusiones sobre la enseñanza del español como lengua materna.

\section{Resultados}

Una vez realizada la investigación, podemos decir que, para lograr una enseñanza efectiva del español como lengua materna en la escuela actual, y darles solución a las deficiencias detectadas por los diferentes estudios; los didactas de la lengua deberán llevar a cabo un análisis pormenorizado de los niveles y planos de la lengua, aun dando por sentado que ello es posible solo desde el punto de vista metodológico.

Cualquiera que sea la estrategia didáctica propuesta por los especialistas de la lengua española debe estar diseñada de acuerdo a la formación real del alumnado, o sea, lo que verdaderamente arroja la práctica diaria durante el proceso de enseñanza - aprendizaje en la escuela; y no con lo concebido en distantes gabinetes burocratizados, ya que es el docente quien conoce las verdaderas dificultades que poseen sus estudiantes en cuanto al dominio del lenguaje.

Cuando los niños se enfrentan al proceso de escritura de aquellos vocablos que escucha a diario, le resulta una tarea de fácil aprensión ya que dominan sin dificultades la mecánica de la vertiente fónica, debido a que nuestro idioma tiene una escritura casi alfabética igual a la fonológica.

La principal tarea del docente durante el desarrollo de la clase de lengua es corregir, a través del método didáctico adecuado, al grupo de estudiantes y hasta donde le sea posible, las conductas fónicas tenidas por estigmatizadas (alternancia $\mathrm{r} / \mathrm{l}$ y viceversa, pérdida de la /d/ intervocálica y de la /d/ y /s/ a final de palabra, con carácter sistemático, etc) y respetar lo legitimado por los programas de estudios de los distintos niveles de enseñanza (seseo, yeísmo, etc), ya que esto está relacionado con la norma lingüística de cada región hispanohablante.

Es por eso que, desde el plano fónico, el docente de la lengua materna tiene que ocuparse y preocuparse por el correcto cumplimiento de las normas ortológicas, puesto que una realidad en la escuela cubana actual es que, a los estudiantes se les dificulta la creación de textos orales en el contexto escolar, ya sea en seminarios, actividades prácticas, exposiciones de temas sobre determinado contenido y otra forma de evaluación oral.

Los profesores se encontrarán y deberán ingeniárselas para resolver aquellos problemas ortográficos derivados de la indistinción fonológica entre b/v - g/j, el yeísmo y/ll, el seseo s/c/z/x y la asimilación total de la /h/. Esta indistinción fónica no se da de igual manera en todas las regiones debido a que esto responde a las variaciones diatópicas de una misma lengua, que 
pueden darse tanto a nivel regional como continental. Es preciso señalar que estos fenómenos no son estigmatizados en la construcción de textos orales, pero conllevan a serios errores ortográficos, si no se es cuidadoso, durante la escritura.

Una vez que sepa escribir, el profesor debe hacer un fuerte énfasis en la enseñanza de la ortografía, sin desdeñar la puntuación debido a las implicaciones sintácticas que tiene esta en la construcción de textos escritos. La revisión que se ha hecho a los bancos de problemas de diversos centros educativos ha arrojado que en lo que respecta a la enseñanza del español como lengua materna, el componente más afectado en la actualidad es el ortográfico y el gramatical.

Es en lo fónico donde más claramente se instituyen las valoraciones sociolingüísticas (neutras, positivas o negativas) es en el seno de la comunidad de hablantes. Por tanto, hay muchos comportamientos fonéticos conceptuados como vulgares dentro de la sociedad (asimilación, elisión, alternancia de /r/, ///, aspiración de /k/, /t/ y /p/) como resultado de las variaciones diatópicas ya mencionadas previamente.

Lamentablemente, la enseñanza del español se ha circunscrito a enseñar los elementos gramaticales como una serie inconexa de categorías con sus conceptos, de forma descriptiva, o sea, se crea un divorcio entre gramática y medio sociocultural; lo que provoca una desmotivación en el alumnado puesto que siempre verán la clase de español como lo mismo.

El enfoque cognitivo, comunicativo y sociocultural propuesto por Roméu (2007) persigue que la enseñanza de la gramática debe esté vinculada a la realidad viva del idioma; el alumno debe ver su lengua materna como algo en constante evolución y al mismo tiempo comprender que él es partícipe de ese proceso evolutivo, tal y como lo fueron sus antecesores. Ya que la frecuencia de uso y la variación contextual, posibilitan la aparición de nuevos significados y nuevas funciones lingüísticas. En relación con lo expuesto Giammatteo (2013) plantea: “... es necesario considerar no solo que las palabras no se dan aisladas, sino que se van integrando en unidades cada vez mayores: sintagmas, oraciones, párrafos, los que a su vez se conectan dentro de una unidad de nivel superior y de naturaleza semántica: el texto. (...) Cada palabra interactúa con otras y se resignifica en función de los diferentes contextos que la encuadran -textual, situacional, históricocultural, etc.-. Lo que da unidad al texto es la intención comunicativa de su productor.” (p. 9).

La ingenuidad de muchos profesores y el mal diagnóstico de su grupo de alumnos no les permite darse cuenta que ahogar al estudiante con insistentes nociones gramaticales de forma descriptiva no lo hace más competente, puesto que lo uno no tiene mucho que ver con lo otro. La competencia lingüística está dada por la capacidad que tenga el alumno de ser un modelo lingüístico en cualquier medio socio - cultural a partir del uso correcto de las estructuras gramaticales que aprenda en la escuela.

El maestro está en la obligación de presentarle al alumno construcciones gramaticales alternativas propias de la lengua culta y literaria, es por ello que tiene tanta importancia la promoción de la lectura y la escritura, ya que la lengua escrita, en sus variadas manifestaciones textuales, refleja la norma culta formal, lo que va a contribuir de forma directa al enriquecimiento del vocabulario del alumnado. Sin embargo, el docente no puede desdeñar la idea de presentar a su grupo de 
estudiantes estructuras que sea propias de la lengua popular pues ese será el mejor camino, para que compruebe las variedades lingüísticas que presenta el español. Solo de esta forma los alumnos podrán pasar del plano idiomático intuitivo al plano idiomático reflexivo.

El nivel léxico, o una buena parte de él, a diferencia de los niveles anteriormente mencionados (el fónico y el morfosintáctico); se considera más extrínseco, mucho más abierto, al depender de factores como la edad, la condición sociocultural, el contexto situacional, la religión, la marginalidad o la integración, la profesión, las aficiones, la ideología, el medio familiar, la concepción del mundo, etc. Por ello, podemos decir que de los niveles abordados en el entorno escolar es el de mayor enriquecimiento; no en vano es donde se encuentra el mayor número de unidades de la lengua. Por tanto, hay que señalar que sin el dominio de una cantidad mínima de clases léxico-sintácticas, no se puede llevar a cabo la comunicación eficaz (proceso que el estudiante desarrolla desde que comienza a hablar).

En este nivel el profesor deberá enfatizar en lo cuantitativo, o sea, tratar que los alumnos afiancen y amplíen su vocabulario tanto activo como pasivo, pero siempre dándole prioridad al léxico frecuente de los alumnos. Se hace necesario crear habilidades en los alumnos para el empleo de forma común de palabras en los diferentes estilos, el profesor para lograr esto se pueden proponer situaciones comunicativas complejas donde el estudiante tenga que hacer uso de las palabras de las que se ha ido haciendo dueño durante el proceso de enseñanza - aprendizaje, o como otra posibilidad de comprobar la aprehensión, podrá someterlos al proceso de construcción de textos en diferentes estilos y contextos comunicativos.

Por tanto, en el proceso de planificación de las clases de lengua, el maestro debe hacer énfasis en aquellos conocimientos que se consideran mínimos pero operativos para el enriquecimiento lexical de los estudiantes: sinonimia, campo semántico, antonimia, derivación, parasíntesis, hiperonimia, acepción, polisemia, solidaridad léxica, homonimia, familia de palabras, fraseología, etc. El docente a medida que el curso escolar vaya en avance podrá comprobar si la capacidad léxica de los alumnos se desarrolla paralela al aumento de sus conocimientos y de sus adquisiciones conceptuales. Esto se materializa de forma muy concreta en el caso de los sustantivos, que representan en nuestra lengua, casi la mitad de las unidades léxicas.

Por lo que se hace necesario poner mayor atención didáctico- lingüístico en aquellas clases de palabras que son más versátiles desde el punto de vista de su utilización: verbos, adjetivos y algunos sustantivos abstractos, incluyendo sus modificaciones derivacionales; a la vez que se fortalezca la conciencia sociolingüística del alumno, haciéndolo reparar en lo adecuado y en lo no adecuado sociocultural y estilísticamente, y facilitándole los recursos lingüístico suficientes para que tenga la capacidad y la madurez lingüística suficiente para evitar en un contexto formal aquello que se considera inapropiado (uso de una palabra tabú, jergal, vulgar, etc.).

En cuanto a qué léxico se debe enseñar o tratar de preservar en el ámbito escolar, Alfaro (2018) en una conferencia sobre Cuestiones de didáctica de la lengua propuso las siguientes recomendaciones:

- Debe hacerle ver a los alumnos que toda palabra, acepción o expresión fijada en la lengua es 
legítima por el solo hecho de existir.

- Conviene promover el uso de todo aquel léxico que, bien por emblemático o por urbano, está legitimado por la norma culta de la comunidad de hablantes.

- Las voces de ámbito de uso general en una comunidad de hablantes, deben considerarse prioritarias frente a aquellas otras de uso más restringido y localizado.

- Se debe estimular el manejo de palabras que supongan algún matiz semántico que no posea ningún aspirante a sustituto del español general.

Además de lo expuesto por Alfaro (2018), no podemos dejar de la mano la incorporación del significado como un aspecto fundamental en el análisis y comprensión de la lengua, ya que esta es la única vía para que la enseñanza del español alcance su verdadera dimensión; así como su valor en la formación de estudiantes lingüísticamente competentes.

Una vez que los docentes sean capaces de enseñar lengua y literatura, a partir de su función en el contexto entonces "... el aprendizaje de la lengua materna deja de ser un procedimiento estéril y mecanicista para convertirse en un espacio para el desarrollo de las habilidades cognitivolingüísticas que los estudiantes necesitan para comprender y producir los textos con los que se vinculan en el proceso de enseñanza - aprendizaje" (Giammatteo, 2013, p.14).

Por lo anteriormente expuesto, los profesores de esta materia deberán considerar lo siguiente:

Comprender las deficiencias que pueda tener el alumnado de forma cabal para poder evitar comportamientos didácticos contraproducentes durante el proceso de enseñanza - aprendizaje y lograr que los estudiantes sean capaces de construir el conocimiento por sí mismos. La mayoría de los niños, cuando ingresan a la escuela, por temprano que lo haga, ya posee dominio de la lengua oral, o por lo menos, de las estructuras básicas que ha aprendido en el ámbito familiar. Por tanto, no tiene sentido la enseñanza del uso oral a partir de cero, puesto que el trae interiorizados una serie de conceptos básicos que le han permitido la comunicación en la familia y el medio social; hacer esto, derivará una serie diversa de problemas.

Es determinante para el alumno la enseñanza de la lecto-escritura, ya que por lo general este saber no se adquiere fuera del contexto escolar. Por tanto, este saber junto con la caligrafía debe ser enseñada desde los primeros años y el maestro primario debe ocuparse de que el estudiante haya vencido los objetivos de esta enseñanza en la asignatura de español; de no ser así esas deficiencias lingüísticas constituirá un serio problema cuando el nivel de saberes vaya en ascenso en las enseñanzas posteriores.

El docente, a partir del diagnóstico de su grupo de estudiantes, puede buscar diversas estrategias didácticas que solucionen los problemas lingüísticos, y generar entre él y el estudiantado, un clima psicológico que no afecte a ese que posee dificultades en el aprendizaje de la lengua.

\section{Conclusiones}


La enseñanza de la lengua va más allá de atiborrar al alumno con conceptos o teoría que muchas veces no comprende, se basa esencialmente en hacerle ver al alumno que la lengua es un organismo vivo que varía de manera constante. Es necesario que los docentes demuestren a sus estudiantes las variaciones diatópicas que puede asumir la lengua en uso, además del papel protagónico que este tiene en el proceso de evolución lingüística.

El uso adecuado de la lengua materna, que forma parte de la identidad de los estudiantes como hispanohablantes, se manifestará a partir de un correcto comportamiento lingüístico, dentro y fuera del centro escolar. Para ello, el docente debe lograr desde la clase de lengua y literatura que el estudiante desarrolle habilidades y destrezas en el manejo del idioma, de este modo convertirse en un modelo lingüístico en cualquier contexto sociocultural.

El proceso de enseñanza - aprendizaje del español como lengua materna se ve afectada a todos los niveles educativos; pues cuando se realiza el análisis de la situación de esta se hace de forma muy somera. Existen dificultades sintácticas, fónicas, morfológicas y léxicas en la enseñanza de nuestro idioma, fundamentalmente en las enseñanzas del nivel medio y medio superior.

Lamentablemente no se está siendo totalmente consecuente con los nefastos resultados que una incorrecta enseñanza del español puede ocasionar en un egresado de cualquiera de estas enseñanzas, y las dificultades que estos tendrán para llevar a cabo una correcta disciplina lingüística.

Para finalizar, se hace necesaria la eliminación durante el proceso de enseñanza - aprendizaje de las barreras que supongan una infravaloración lingüística de los alumnos, ya que el docente debe trabajar sobre la base de las verdaderas deficiencias que detecte durante la realización del diagnóstico en su grupo de estudiantes, y a partir de ahí trazarse una serie de estrategias didácticas y metodológicas para darle solución, teniendo en cuenta sus intereses como el ser social hispanohablante que es. 


\section{Referencias}

Addine, F., Recarey, S., Fuxá, M., \& Fernández, S. (2004). Didáctica: teoría y práctica. La Habana: Editorial Pueblo y Educación, 51.

Alfaro, L. (2018). El español actual y sus problemas: Cuestiones de didáctica de la lengua. [diapositiva de Power Point]. UCLV. Villa Clara, Cuba.

Bruzual, R. (2007). Fundamentos teóricos y metodológicos para la enseñanza de la lengua materna (L1) y segundas lenguas (L2) en contextos bilingües. Argos, 24(46), 46-65.

Cassany, D., Luna, M., \& Sanz, G. (1994). Enseñar lengua. Barcelona: Graó.

Giammatteo, M. (diciembre, 2013). ¿Por qué y para qué enseñar gramática? La gramática en la formación de habilidades cognitivo lingüísticas. SIGNOS ELE, 7, URL http://p3.usal.edu.ar/index.php/ele/article/ view/2003, 16 pp.

Hernández, R., Fernández, C., \& Baptista, M. (2014). Metodología de la investigación. Iztapalapa. México D.F., México: Mc Graw Hill

Lomas, C. (1999). Cómo enseñar a hacer cosas con las palabras: teoría y práctica de la educación lingüística. Barcelona: Paidós.

Ministerio de Educación, Cultura y Deporte (1997). Currículo Básico Nacional. Caracas, Venezuela: UCEP

Parra, M. (1989). Español comunicativo. Universidad nacional de Colombia. Colombia: Editorial Norma S.A

Roméu Escobar, A. (1992). Aplicación del enfoque comunicativo: comprensión, análisis y construcción de textos. Ciudad Habana: IPLAC.

Roméu, A. (2003). Acerca de la enseñanza del español y la Literatura. Pueblo y Educación, 20-24.

Roméu, A. (2007). El enfoque cognitivo, comunicativo y sociocultural en la enseñanza de la lengua y la literatura. La Habana: Editorial Pueblo y Educación, 112-29.

Roméu, A. (2013). Didáctica de la lengua española y la literatura. La Habana: Editorial Pueblo y Educación.

Saussure, F. (1975). Curso de Lingüística general. Madrid, España: Losada 
Copyright (c) 2020 Armando Castillo Acevedo y Heriberto Nuñez Rodríguez

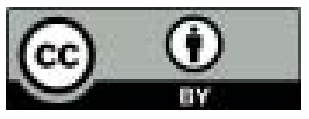

Este texto está protegido bajo una licencia internacional Creative Commons 4.0.

Usted es libre para Compartir-copiar y redistribuir el material en cualquier medio o formato - y Adaptar el documento - remezclar, transformar y crear a partir del material-para cualquier propósito, incluso para fines comerciales, siempre que cumpla las condiciones de Atribución. Usted debe dar crédito a la obra original de manera adecuada, proporcionar un enlace a la licencia, e indicar si se han realizado cambios. Puede hacerlo en cualquier forma razonable, pero no de forma tal que sugiera que tiene el apoyo del licenciante o lo recibe por el uso que hace de la obra.

\section{$\underline{\text { Resumen de licencia - Texto completo de la licencia }}$}

